\title{
Can metformin stabilize PCSK9 level in stable coronary artery disease patients treated with statins?
}

\author{
Aleksandr B. Shek, Rano B. Alieva, Ravshanbek D. Kurbanov, Shavkat U. Hoshimov,
}

Ulugbek I. Nizamov, Adolat V. Ziyaeva

Department of Ischemic Heart Disease and Atherosclerosis, Republican Specialised Center of Cardiology, Tashkent, Uzbekistan

Submitted: 21 March 2019

Accepted: 2 June 2019

Arch Med Sci Atheroscler Dis 2019; 4: e144-e150

DOI: https://doi.org/10.5114/amsad.2019.86752

Copyright (c) 2019 Termedia \& Banach

\section{Abstract}

Introduction: Proprotein convertase subtilisin/kexin type 9 (PCSK9) has emerged as an important marker of cardiovascular risk and a new target for therapeutic interventions. We aimed to study the influence of metformin on the level of circulating PCSK9 in patients with stable coronary artery disease (SCAD) and type 2 diabetes (T2DM) or metabolic syndrome (MetS), receiving moderate doses of statins used in routine clinical practice.

Material and methods: The study included 80 patients with T2DM or MetS receiving rosuvastatin for at least three months prior the study. MetS was diagnosed based on the Global Consensus Definition of the International Diabetes Federation (IDF). Serum level of PCSK9 was measured with an ELISA kit. Results: Patients with T2DM or MetS, who took part in the research, were divided into 2 groups - those who received metformin prior the main study (21 patients $-1^{\text {st }}$ group) and patients who did not (59 patients $-2^{\text {nd }}$ group). Addition of metformin to the 3-month statin therapy of the 2 nd group patients, divided into subgroup $\mathrm{A}(n=27)$ with the addition of metformin and subgroup $B(n=29)$ without one, did not significantly affect the level of lipids. However, the level of circulating PCSK9 in subgroup A patients decreased, compared to subgroup B $(p<0.01)$. At the same time, ongoing metformin and rosuvastatin therapy in the $1^{\text {st }}$ group patients was not accompanied by a further decrease of the PCSK9 level.

Conclusions: The addition of metformin to ongoing rosuvastatin therapy did not significantly affect serum lipid levels, but stabilized the level of circulating PCSK9, compared with the group without metformin treatment.

Key words: metformin, rosuvastatin, proprotein convertase subtilisin/kexin type 9 , type 2 diabetes mellitus, metabolic syndrome.

\section{Introduction}

Statin treatment is a cornerstone of cardiovascular disease prevention and treatment [1-3]. However, by lowering low-density lipoprotein cholesterol (LDL-cholesterol) in the blood, statins activate the expression of the proprotein convertase subtilisin/kexin type 9 (PCSK9), which is a secreted LDL receptor inhibitor, and a risk predictor of cardiovascular events [4]. Perhaps that is why application of maximal doses of statins during the therapy does not prevent residual risk of cardiovascular events and cannot lower LDL cholesterol to the target level [5]. Also, it has been shown that moderate statin therapy (atorvastatin $40 \mathrm{mg}$ ) causes an increase in PCSK9 levels after 16 weeks [6]. The connection between

\author{
Corresponding author: \\ Prof. Aleksandr B. Shek \\ Republican Specialised \\ Center of Cardiology \\ Tashkent, Uzbekistan \\ Phone: +998712525455 \\ E-mail: shek-999@mail.ru
}


the level of PCSK9 and the risk of major adverse cardiovascular events (MACE) has been proven in a number of studies [7-9].

In this regard, nowadays there are two main targets for atherosclerotic cardiovascular disease (ASCVD): LDL cholesterol and PCSK9 [4, 10]. Monoclonal antibodies (MAB) which target PCSK9 lower LDL cholesterol (LDL-C) levels by $55 \%$ to $72 \%$ in high-risk patient groups and are the only tool which effectively reduces both LDL-C and PCSK9 $[11,12]$. Unfortunately, the use of $M A B$ in some cases is likely to be limited by cost factors.

During the Dallas Heart Study - a large multi-ethnic study $(n=3138)$ - Lakoski et al. [13] found that PCSK9 levels correlated significantly $(p<0.001)$ with fasting serum glucose, insulin, homeostatic model assessment of insulin resistance (HOMA-IR), obesity and diabetes. Another study, which included patients with type 2 diabetes (T2DM) and other metabolic disorders (metabolic syndrome (MetS), obesity and atherogenic dyslipidemia), showed that PCSK9 levels are increased in patients with T2DM and MetS and correlated with an atherogenic lipid profile and insulin resistance parameters [14]. Although precise mechanisms of PCSK9 regulation by insulin are not completely understood, a review of the literature shows that insulin regulates PCSK9 through a dual effect - induction and/or inhibition [15]. It is also well known that metformin is the best choice for reducing the main components of the metabolic syndrome - it reduces weight loss, improves the lipid profile and insulin resistance, and decreases the incidence of T2DM by $40 \%[16,17]$.

Considering all the above, we aimed to study the influence of metformin on the level of circulating PCSK9 in patients with stable coronary artery disease (SCAD) and T2DM or MetS, receiving moderate doses of statins used in routine clinical practice.

\section{Material and methods}

The study was carried out on 80 patients who were hospitalized in the Department of Ischemic Heart Disease and Atherosclerosis of the Republican Specialized Center of Cardiology from November 2017 to July 2018 and who were diagnosed with chronic stable coronary artery disease (SCAD) on discharge. The protocol of the study was approved by the Ethics Committee under the Republican Specialized Center of Cardiology.

For the study only patients were taken:

- With diagnoses T2DM, who, prior to randomization, did not take hypoglycemic drugs other than metformin and/or sulfonylureas and MetS, who took or did not take metformin;

- Who took a course of statins (rosuvastatin 10$20 \mathrm{mg} /$ day) for at least 3 months prior the study.
MetS was diagnosed based on the Global Consensus Definition of the International Diabetes Federation (IDF) [18]. This definition identified central obesity as an essential component of MetS and defined MetS as a cluster of the most dangerous heart attack risk factors which include central obesity (abdominal obesity as $\geq 94 \mathrm{~cm}$ in men or $\geq 80 \mathrm{~cm}$ in women) plus any two of four parameters, based on increased triglycerides, lower low-density lipoprotein (HDL) cholesterol, high blood pressure and fasting plasma glucose levels [18].

Exclusion criteria were: New York Heart Association functional class 3 or 4 heart failure; uncontrolled angina; hepatic impairment (based on assessment of liver function tests and liver imaging); renal impairment (estimated glomerular filtration rate $<45 \mathrm{ml}$ $\mathrm{min} / 1.73 \mathrm{~m}^{2}$ ); acute illness (temperature, dehydration, severe infection, acute cardiac failure).

Depending on the metformin administration, all patients were divided into 2 groups: group I - patients who took extended-release metformin (dose of 500-1000 mg/day) before dividing into groups $(n=21)$; group $\|$ - patients who did not take metformin before the study $(n=59)$. After the baseline examination, patients of group I continued to receive rosuvastatin and metformin treatment for 3 months. In group II, all the patients were equally divided into 2 subgroups in an open-label crossover study protocol. Subgroup A consisted of 30 SCAD and T2DM or MetS patients who were treated with rosuvastatin 10-20 mg/day and metformin at a dose of $500 \mathrm{mg} /$ day, and subgroup B had 29 patients who continued treatment with rosuvastatin $10-20 \mathrm{mg} /$ day without metformin. However, in subgroup A, after addition of metformin to the treatment course, 3 patients suffered from gastrointestinal disorders (diarrhea); therefore, metformin was excluded from the treatment. Therefore, in subgroup $A$ the number of patients was 27 , in subgroup $B$ it was 29. After 2 weeks in subgroup $A$, in 27 patients with good tolerability of metformin, its dose was increased to $1000 \mathrm{mg}$ per day, but 3 patients had dyspeptic disorders (diarrhea, nausea or vomiting) and returned to a dose of $500 \mathrm{mg} /$ day.

The basic therapy included antiplatelets (100\%), $\beta$-blockers (100\%), if required, renin-angiotensin system (RAS) blockers (90\%) and short-acting nitrates. As noted above, the study did not include patients who took other hypoglycemic drugs other than metformin, with the exception of sulphonylureas, which, as has been proven $[19,20]$, do not improve insulin resistance, lipid metabolism and the prognosis of patients.

\section{Functional and biochemical studies}

To verify the $\mathrm{CHD}$ diagnosis, the following tests were performed: 12-lead ECG, echocardiography 
(EchoCG), 24-h Holter ECG monitoring, exercise stress test and coronary angiography, if required. Carotid artery ultrasound scans were performed at baseline examination and after 3 months with a SIEMENS Acuson X700. The following blood lipid spectrum parameters were studied: total cholesterol (TC), low-density lipoprotein cholesterol (LDL-C), high-density lipoprotein cholesterol (HDL-C), triglycerides (TG), very low-density lipoprotein cholesterol (VLDL-C), high-sensitive C-reactive protein (hsCRP), on a Daytona autoanalyzer (Randox, Ireland). The level of PCSK9 in the blood was measured with the enzyme-linked immunoassay, using a commercially available kit: Human Proprotein Convertase 9/PCSK9 ELISA Kit (MULTI SCIENCE, China).

\section{Statistical analysis}

Statistical analysis of the data was performed using Statistica 10.0. Obtained data were presented as mean and standard deviation ( $m \pm$ $\mathrm{SD})$, where the statistical significance of the obtained measurements for compared mean values was determined by Student's $t$-test $(t)$ with error probability $(p)$ calculated to check normality of the distribution. In particular, independent samples Student's $t$-test was used for between-group analysis and paired samples Student's $t$-test was used for repeated measures analysis. If the distribution of studied variables differed from the normal distribution, the non-parametric analysis tests, Wilcoxon signed-rank, to compare two dependent samples, matched samples, or repeated measurements, Mann-Whitney T-test for two independent groups and Kruskal-Wallis ANOVA for multiple independent groups were used. In order to find differences between qualitative statistical measures, the $\chi^{2}$ method was used together with Fisher's exact test for small samples.

\section{Results}

Among the examined 80 patients with T2DM or MetS, 21 patients (group I) took metformin (dose of 500-1000 mg/day) before the beginning of the observation. It was noted that patients of group I (Table I) more commonly had cases of diabetes mellitus $(p<0.001)$, myocardial infarction ( $p<0.001)$, stroke $(p<0.05)$ and percutaneous coronary intervention with a stenting history $(p<$ $0.05)$ compared to the patients of group II $(n=59)$. This was accompanied by an increased fasting glucose level $(p<0.01)$ (Table II) and an increase in the thickness of the carotid intima-media complex on the left and right $(p<0.01)$ in patients of group I, compared to patients of group II $(p<0.05)$ (Table I).

The level of lipids of patients of the two groups did not significantly differ (Table II), as all of the patients took rosuvastatin $10-20 \mathrm{mg} /$ day, at least
3 months before the start of study (Table I). However, despite the predominance of diabetes mellitus and MACE in group I patients, the average level of their circulating PCSK9 was not higher (Table II) compared to group II patients (581 vs. $658 \mathrm{ng} / \mathrm{ml}, p=0.10)$.

After baseline examination, as mentioned above, patients of group II were equally divided into 2 subgroups - IIA (basic treatment + metformin) and IIB (continued basic treatment without metformin) - but 3 patients were excluded from the study due to intolerance of metformin. The subgroups did not differ in the main clinical parameters, structural changes of carotid arteries, lipid levels and hsCRP (Tables I, II). In subgroup A there were $9(33 \%)$ patients with diabetes mellitus, whereas in B only 5 (17\%). The level of PCSK9 in subgroups IIA and IIB was 772 vs. 602, respectively $(p=0.10)$.

Analysis of the dynamics, after three months of observation, showed that the level of lipids did not decrease further in both groups I and II (in both subgroups of group II as well) (Table II). There was no difference in glucose levels after addition of metformin to the therapy of patients in subgroup A (from initial $=6.7 \pm 1.8 \mathrm{mg} / \mathrm{dl}$ to $6.0 \pm 2.0$ $\mathrm{mg} / \mathrm{dl})$ after 3 months $(p=0.18)$.

Ongoing rosuvastatin therapy in group I, did not significantly increase the level of PCSK9, but the level of PCSK9 in patients of group II was different: in subgroup A it decreased significantly compared to the initial level $(p<0.001)$, whereas in subgroup B it increased $(p<0.01)$. As a result, addition of metformin to rosuvastatin treatment was accompanied by a decrease in the level of PCSK9 in subgroup A compared to subgroup B $(p<0.01)$, after 3 months of therapy.

\section{Discussion}

Our results showed that the level of circulating PCSK9 is not significantly distinguished among the patients of both groups with SCAD and DM or MetS, receiving metformin in addition to rosuvastatin (group I), or not receiving it (rosuvastatin only) (group II). However, there were significantly more cases of diabetes mellitus, myocardial infarction, stroke and percutaneous coronary intervention among the patients of group I, compared to group II, which in some cases could be accompanied by a higher level of PCSK9. The correlation between an increase in the level of circulating PCSK9 and the risk of a MACE had been shown in a number of recent studies [21, 22]. In particular, PCSK9 level was associated with insulin levels and HOMA-IR index in patients with stable CAD on statin treatment [7]. The results of the HUNT study in Norway [8] showed that a high level of PCSK9 was positively associated with risk of $\mathrm{MI}$ among 
Table I. Baseline clinical hemodynamic and biochemical parameters of group I and II patients (M \pm SD, $n(\%))$

\begin{tabular}{|c|c|c|c|c|c|}
\hline Parameters & All $(n=80)$ & $\begin{array}{l}\text { Group I, met- } \\
\text { formin prior the } \\
\text { study }(n=21)\end{array}$ & $\begin{array}{l}\text { Group II, } \\
\text { absence of met- } \\
\text { formin prior the } \\
\text { study }(n=59)\end{array}$ & $\begin{array}{l}\text { IIA, with } \\
\text { metformin } \\
(n=27)^{\#}\end{array}$ & $\begin{array}{c}\text { IIB, without } \\
\text { metformin } \\
(n=29)\end{array}$ \\
\hline Average age [years] & $61.3 \pm 9.5$ & $62.0 \pm 11.7$ & $61.8 \pm 8.8$ & $63.1 \pm 8.0$ & $59.7 \pm 8.9$ \\
\hline Sex (male/female) & $\begin{array}{c}34 / 46 \\
(43 \% / 57 \%)\end{array}$ & $\begin{array}{c}8 / 13 \\
(38 \% / 62 \%)\end{array}$ & $\begin{array}{c}26 / 33 \\
(44 \% / 56 \%)\end{array}$ & $\begin{array}{c}14 / 13 \\
(52 \% / 48 \%)\end{array}$ & $\begin{array}{c}12 / 17 \\
(41 \% / 59 \%)\end{array}$ \\
\hline $\mathrm{BMI}\left[\mathrm{kg} / \mathrm{m}^{2}\right]$ & $29.0 \pm 4.9$ & $28.6 \pm 3.0$ & $29.7 \pm 4.4$ & $30.4 \pm 3.8$ & $28.7 \pm 5.0$ \\
\hline $\begin{array}{l}\text { Arterial } \\
\text { hypertension (\%) }\end{array}$ & 100 & 100 & 100 & 100 & 100 \\
\hline $\begin{array}{l}\text { Diabetes mellitus, } \\
n(\%)\end{array}$ & $29(36)$ & $15(71)^{\star * *}$ & $14(24)$ & $9(33)$ & $5(17)$ \\
\hline $\begin{array}{l}\text { History of } \\
\text { myocardial } \\
\text { infarction, } n(\%)\end{array}$ & $29(36)$ & $14(67)^{\star \star *}$ & $15(25)$ & $6(22)$ & $8(28)$ \\
\hline $\begin{array}{l}\text { History of stroke, } \\
n(\%)\end{array}$ & $4(5)$ & $3(14)^{*}$ & $1(2)$ & $1(4)$ & - \\
\hline $\begin{array}{l}\text { Aortocoronary } \\
\text { bypass grafting, } \\
n(\%)\end{array}$ & $8(10)$ & $4(19)$ & $4(5)$ & $2(11)$ & $2(6)$ \\
\hline $\begin{array}{l}\text { Percutaneous } \\
\text { coronary } \\
\text { intervention, } n(\%)\end{array}$ & $19(24)$ & $9(43)^{*}$ & $10(17)$ & $4(15)$ & $5(17)$ \\
\hline CIMT left [mm] & $1.11 \pm 0.17$ & $1.24 \pm 0.15^{\star \star}$ & $1.04 \pm 0.21$ & $1.10 \pm 0.19$ & $1.00 \pm 0.20$ \\
\hline CIMT right $[\mathrm{mm}]$ & $1.10 \pm 0.12$ & $1.22 \pm 0.08^{\star}$ & $1.03 \pm 0.18$ & $1.09 \pm 0.18$ & $1.00 \pm 0.19$ \\
\hline $\begin{array}{l}\text { Presence of } \\
\text { atherosclerotic } \\
\text { plaque on the left } \\
(C C A, I C A), n(\%)\end{array}$ & $38(48)$ & $10(48)$ & $28(48)$ & $11(41)$ & $16(55)$ \\
\hline $\begin{array}{l}\text { Presence of } \\
\text { atherosclerotic } \\
\text { plaque on the right } \\
\text { (CCA, ICA), } n(\%)\end{array}$ & $33(41)$ & $8(38)$ & $25(42)$ & $11(41)$ & $14(45)$ \\
\hline $\begin{array}{l}\% \text { stenosis on the } \\
\text { left (CCA, ICA) }\end{array}$ & 32.5 & 32.8 & 32.0 & 30.8 & 32.9 \\
\hline $\begin{array}{l}\% \text { stenosis on the } \\
\text { right (CCA, ICA) }\end{array}$ & 33.1 & 35.3 & 31.6 & 35.9 & 31.3 \\
\hline $\begin{array}{l}\text { Rosuvastatin } \\
\text { average dose (dose } \\
\text { range) }[\mathrm{mg} / \text { day] }\end{array}$ & $16.3(10-20)$ & $16.7(10-20)$ & $16.1(10-20)$ & $16.3(10-20)$ & $16.2(10-20)$ \\
\hline $\begin{array}{l}\text { Rosuvastatin } \\
\text { treatment before } \\
\text { the study [months] }\end{array}$ & $5.5 \pm 1.5$ & $5.8 \pm 1.8$ & $5.4 \pm 1.6$ & $5.3 \pm 1.4$ & $5.4 \pm 1.7$ \\
\hline $\begin{array}{l}\text { Sulfonylurea drugs, } \\
n(\%)\end{array}$ & $21(26)$ & $7(33)$ & $14(24)$ & $9(33)$ & $5(17)$ \\
\hline
\end{tabular}

Values are summarized as mean $\pm S D$, median (interquartile range) and categorical variables as percentage; "3 patients excluded due to intolerance to metformin; ${ }^{*} p<0.05,{ }^{* *} p<0.01,{ }^{* * *} p<0.001$ significance of the difference between groups I and II. CIMT - carotid intimamedia thickness, CCA - common carotid artery, ICA-internal carotid artery.

1,488 patients with a first incident of $\mathrm{MI}$, and the level of serum PCSK9 was an independent predictor of MACE (death, acute coronary syndrome, and cerebral stroke) in 306 patients with SCAD [9]. On the other hand, the FOURIER randomized controlled trial had shown [23] that the risk of primary endpoints (cardiovascular death, myocardial infarction, stroke, hospital admission for unstable angina, or coronary revascularization) of diabetic patients was decreased by $17 \%$ by reducing the level of PCSK9.

The classic UKPDS study (United Kingdom Prospective Diabetes Study), which lasted 11 years, showed for the first time that metformin, regard- 
Table II. Dynamics (3 months) of blood lipids, hsCRP and circulating PCSK9 in the studied groups* $(M \pm S D, n(\%))$

\begin{tabular}{|c|c|c|c|c|c|c|c|}
\hline \multirow[t]{3}{*}{ Parameter } & \multirow{2}{*}{\multicolumn{2}{|c|}{$I(n=21)$}} & \multirow{3}{*}{$\begin{array}{l}\text { II }(n=59) \\
\text { Baseline }\end{array}$} & \multicolumn{4}{|c|}{ II, dynamics in subgroups } \\
\hline & & & & \multicolumn{2}{|c|}{ II A $(n=27)^{\#}$} & \multicolumn{2}{|c|}{ II B $(n=29)$} \\
\hline & Baseline & 3 months & & Baseline & 3 months & Baseline & 3 months \\
\hline $\begin{array}{l}\text { Total cholesterol } \\
\text { (CS) }[\mathrm{mg} / \mathrm{dl}]\end{array}$ & $166.6 \pm 40.3$ & $158.0 \pm 37.7$ & $178.3 \pm 37.5$ & $182.2 \pm 43.5$ & $176.9 \pm 39.7$ & $173.5 \pm 40.7$ & $162.0 \pm 46.7$ \\
\hline TG [mg/dl] & $\begin{array}{c}189.5 \\
(115-318)\end{array}$ & $\begin{array}{c}149.5 \\
(83-185)\end{array}$ & $\begin{array}{c}155.5 \\
(109-215)\end{array}$ & $\begin{array}{c}160.0 \\
(117-230)\end{array}$ & $\begin{array}{c}132.0 \\
(114-139)\end{array}$ & $\begin{array}{c}152.5 \\
(104-208)\end{array}$ & $\begin{array}{c}126 \\
(91-174)\end{array}$ \\
\hline $\mathrm{HDL}-\mathrm{C}[\mathrm{mg} / \mathrm{dl}]$ & $39.2 \pm 9.0$ & $40.8 \pm 12.1$ & $43.7 \pm 8.7$ & $44.3 \pm 9.3$ & $44.6 \pm 11.6$ & $43.6 \pm 9.0$ & $43.7 \pm 9.5$ \\
\hline LDL-C [mg/dl] & $82.2 \pm 39.9$ & $67.8 \pm 26.4$ & $101.9 \pm 39.2$ & $101.2 \pm 33.4$ & $101.1 \pm 33.9$ & $94.5 \pm 36.3$ & $88.4 \pm 36.8$ \\
\hline $\begin{array}{l}\text { Glucose } \\
{[\mathrm{mmol} / \mathrm{l}]}\end{array}$ & $8.6 \pm 2.1^{* *}$ & $7.9 \pm 2.5$ & $6.5 \pm 1.6$ & $6.7 \pm 1.8$ & $6.0 \pm 2.0$ & $6.5 \pm 1.9$ & $6.3 \pm 2.3$ \\
\hline $\begin{array}{l}\text { C-reactive } \\
\text { protein (CRP) } \\
{[\mathrm{mg} / \mathrm{l}]}\end{array}$ & $\begin{array}{c}6.9 \\
(3.0-8.5)\end{array}$ & $\begin{array}{c}5.4 \\
(4.5-7.4)\end{array}$ & $\begin{array}{c}5.9 \\
(3.2-7.1)\end{array}$ & $\begin{array}{c}6.0 \\
(4.7-7.1)\end{array}$ & $\begin{array}{c}6.6 \\
(4.4-8.3)\end{array}$ & $\begin{array}{c}5.5 \\
(2.1-7.5)\end{array}$ & $\begin{array}{c}5.5 \\
(2.2-7.5)\end{array}$ \\
\hline PCSK9 [ng/ml] & $\begin{array}{c}581 \\
(450-697)\end{array}$ & $\begin{array}{c}618 \\
(568-703)\end{array}$ & $\begin{array}{c}658 \\
(516-814)\end{array}$ & $\begin{array}{c}772 \\
(526-1083)\end{array}$ & $\begin{array}{c}537 \\
(405-676) \\
* *(* * *)\end{array}$ & $\begin{array}{c}602 \\
(516-706)\end{array}$ & $\begin{array}{c}787 \\
(667-989) \\
\left({ }^{* *}\right)\end{array}$ \\
\hline
\end{tabular}

Values are summarized as mean $\pm S D$, median (interquartile range) and categorical variables as percentage. ${ }^{*}$ Group $I-m e t f o r m i n$ administered prior the study $(n=21)$; II, without metformin prior the study $(n=59)$; / $A$ - metformin added $(n=27) ; \| B-n o$ added metformin $(n=29)$. " 3 patients excluded due to intolerance to metformin; ${ }^{* *} p<0.01$ - significance of the difference between the subgroups $A$ and $B ;{ }^{* *},{ }^{* *} p<0.01, p<0.001-$ significance of the difference compared to the baseline level.

less of its hypoglycemic action, compared to sulfonylurea and insulin, causes a statistically significant reduction of overall mortality, mortality from diabetes and mortality from myocardial infarction [19]. Treatment with metformin reduces insulin resistance in diabetic patients and therefore reduces the severity of its atherogenic effects [24]. Some studies have shown that metformin treatment reduces the severity of atherogenic dyslipidemia [25]. At the same time, a recent study conducted in Glasgow ("the CAMERA study") showed that addition of metformin to statins in moderate, not intensive doses, had no effect on CIMT and such parameters as total cholesterol, HDL cholesterol, non-HDL cholesterol, triglycerides, hsCRP, or fasting glucose, but at the same time reduced a number of other important surrogate markers $\left(\mathrm{HbA}_{1 \mathrm{c}}\right.$, insulin, HOMA-IR, and tissue plasminogen activator) of cardiovascular disease in non-diabetic patients with high cardiovascular risk [26].

In our study it was also found that addition of metformin to the 3-month statin therapy of group II patients did not significantly affect the level of lipids. However, the level of circulating PCSK9 in subgroup A decreased after 3 months of treatment, compared to subgroup B. At the same time, ongoing metformin treatment in group I patients was not accompanied by a further decrease in the PCSK9 concentration, which possibly testifies about stabilization of its level, instead of the increase, usually caused by statin treatment.

As mentioned above, a number of studies have shown the correlation between insulin resistance, impaired glucose tolerance, diabetes mellitus and increased level of blood PCSK9 [14, 27-30], while in others, although this correlation was not confirmed, the role of PCSK9 in dyslipidemia modification was demonstrated [31]. It is known that hepatic PCSK9 expression is regulated by insulin via the sterol regulatory element binding protein I-C (SREBP-1C); thus, PCSK9 is secreted in an insulin-dependent fashion [32], which explains the association of PCSK9 with MetS [33, 34].

Metformin also activates AMPK in hepatocytes, which leads to a decrease in production of fatty acids and a consequent decrease in lipidemia and hepatic steatosis and an increase in the insulin sensitivity of liver cells [35]. Metformin increases activity of AMPK in skeletal muscles, which promotes greater uptake of glucose during contractile processes [36].

It is well known that only MAB, which target PCSK9 in high-risk patient groups, are the only tool which effectively reduces both LDL-C and PCSK9. However, since PCSK9 is involved in insulin-related pathophysiologies, such as MetS and T2DM, the results of research showing that some drugs that can reduce insulin resistance, such as liraglutide, a glucagon-like peptide-1 receptor agonist, can, to various extents, affect PCSK9 metabolism are interesting [37]. Also, recent studies on primary mouse and human hepatocytes provide partial support for the concept that metformin and AMPK activation may provide cardiovascular benefit via increased reverse cholesterol transport, induction of Abcg 5 and Abcg8 expression, and indicate increased LDLR expression as a potential additional mechanism [38]. 
Considering the above, we can only suggest that the addition of metformin to treatment can reduce the excessive increase of PCSK9 usually caused by statins (one of the possible mechanisms is insulin resistance). After that, it does not affect the basal secretion level of PCSK9 (initially high or (ow), and therefore it is unpromising in some categories of patients (for example, in familial heterozygous hypercholesterolemia).

The present study has several limitations. The first is the relatively small number of patients, associated with the fact that the study included patients with SCAD and T2DM or MetS who, prior to randomization, did not take hypoglycemic drugs other than metformin and/or sulfonylureas. Secondly, all patients took rosuvastatin in doses that were lower than recommended high-intensity statin therapy. Further research is needed, which should be carried out on a large number of observations.

In conclusion, the addition of metformin to ongoing rosuvastatin therapy did not significantly affect serum lipid levels, but stabilized the level of circulating PCSK9, compared with those without metformin treatment. Ongoing metformin treatment in SCAD patients who took rosuvastatin and metformin prior the study was not accompanied by a further decrease in the PCSK9 concentration, which indirectly indicates only the stabilization of its level instead of the increase usually caused by statin treatment.

\section{Acknowledgments}

The study was supported by a research grant from the Ministry of Innovation Development of the Republic of Uzbekistan (Contract No PZ20170912103).

\section{Conflict of interest}

The authors declare no conflict of interest.

\section{References}

1. Catapano AL, Graham I, De Backer G, et al. 2016 ESC/ EAS guidelines for the management of dyslipidaemias: the Task Force for the management of dyslipidaemias of the European Society of Cardiology (ESC) and the European Atherosclerosis Society (EAS). Eur Heart J 2016; 37: 2999-3058

2. Baigent C, Keech A, Kearney PM, et al.; Cholesterol Treatment Trialists' (CTT) Collaborators. Efficacy and safety of cholesterol-lowering treatment: prospective meta-analysis of data from 90,056 participants in 14 randomised trials of statins. Lancet 2005 ; 366: 1267-78.

3. Ford ES, Ajani UA, Croft JB, et al. Explaining the decrease in U.S. deaths from coronary disease, 1980-2000. N Engl J Med 2007; 356: 2388-98.

4. Seidah NG, Awan Z, Chrétien M, Mbikay M. PCSK9: a key modulator of cardiovascular health. Circ Res 2014; 114: 1022-36.
5. Waters DD, Brotons C, Chiang CW, et al. Lipid Treatment Assessment Project 2 Investigators; Lipid treatment assessment project 2: a multinational survey to evaluate the proportion of patients achieving low-density lipoprotein cholesterol goals. Circulation 2009; 120: 28-34.

6. Careskey HE, Davis RA, Alborn WE, Troutt JS, Cao G, Konrad RJ. Atorvastatin increases human serum levels of proprotein convertase subtilisin/kexin type 9. J Lipid Res 2008; 49: 394-8.

7. Werner C, Hoffmann MM, Winkler K, Böhm M, Laufs U. Risk prediction with proprotein convertase subtilisin/ kexin type 9 (PCSK9) in patients with stable coronary disease on statin treatment. Vascul Pharmacol 2014; 62: 94-102.

8. Laugsand LE, Åsvold BO, Vatten LJ, et al. Circulating PCSK9 and risk of myocardial infarction: the HUNT Study in Norway. JACC Basic Transl Sci 2016; 1: 568-75.

9. Sakamoto A, Nobukazu I, Uehara M, Ando J, Komuro I. Elevated serum proprotein convertase subtilisin/kexin type 9 level independently predicts long-term cardiovascular outcomes in patients with chronic coronary artery disease. Circulation 2018; 134: A13236.

10. Banach M, Rizzo M, Obradovic M, et al. PCSK9 inhibition - a novel mechanism to treat lipid disorders? Curr Pharm Des 2013; 19: 3869-77.

11. Annemans L, Packard CJ, Briggs A, Ray KK. "Highest risk-highest benefit" strategy: a pragmatic, cost-effective approach to targeting use of PCSK9 inhibitor therapies. Eur Heart J 2018; 39: 2546-50.

12. Rosenson RS, Hegele RA, Fazio S, Cannon CP. The evolving future of PCSK9 inhibitors. J Am Coll Cardiol 2018; 72: 314-29.

13. Lakoski SG, Lagace TA, Cohen JC, Horton JD, Hobbs HH. Genetic and metabolic determinants of plasma PCSK9 levels. J Clin Endocrinol Metab 2009; 94: 2537-43.

14. Ibarretxe D, Girona J, Plana N, et al. Circulating PCSK9 in patients with type 2 diabetes and related metabolic disorders. Clin Investig Arterioscler 2016; 28: 71-8.

15. Momtazi AA, Banach M, Pirro M, Stein EA, Sahebkar A. PCSK9 and diabetes: is there a link? Drug Discov Today 2017; 22: 883-95.

16. Salpeter SR, Buckley NS, Kahn JA, et al. Meta-analysis: metformin treatment in persons at risk for diabetes mellitus. Am J Med 2008; 121: 149-57e2.

17. Ladeiras-Lopes R, Fontes-Carvalho R, Bettencourt N, Sampaio F, Gama V, Leite-Moreira A. Novel therapeutic targets of metformin: metabolic syndrome and cardiovascular disease. Expert Opin Ther Targets 2015; 19: 869-77.

18. Alberti KG, Zimmet P, Shaw J. IDF Epidemiology Task Force Consensus Group. Metabolic syndrome - a new worldwide definition. Lancet 2005; 366: 1059-62.

19. UK Prospective Diabetes Study (UKPDS) Group. Intensive blood-glucose control with sulphonylureas or insulin compared with conventional treatment and risk of complications in patients with type 2 diabetes (UKPDS 33). Lancet 1998; 352: 837-53.

20. Ferrannini E, DeFronzo RA. Impact of glucose-lowering drugs on cardiovascular disease in type 2 diabetes. Eur Heart J 2015; 36: 2288-96.

21. Vlachopoulos C, Terentes-Printzios D, Georgiopoulos G, et al. Prediction of cardiovascular events with levels of proprotein convertase subtilisin/kexin type 9: a systematic review and meta-analysis. Atherosclerosis 2016; 252: 50-60.

22. El Khoury P, Roussel R, Fumeron F, et al. Plasma proprotein-convertase-subtilisin/kexin type 9 (PCSK9) and 
cardiovascular events in type 2 diabetes. Diabetes Obes Metab 2018; 20: 943-53.

23. Sabatine MS, Leiter LA, Wiviott SD, et al. Cardiovascular safety and efficacy of the PCSK9 inhibitor evolocumab in patients with and without diabetes and the effect of evolocumab on glycaemia and risk of new-onset diabetes: a prespecified analysis of the FOURIER randomized controlled trial. Lancet Diabetes Endocrinol 2017; 5: 941-50.

24. Libby P, Plutzky J. Diabetic macrovascular disease: the glucose paradox? Circulation 2002; 106: 2760-3.

25. Despres JP. Potential contribution of metformin to the management of cardiovascular disease risk in patients with abdominal obesity, the metabolic syndrome and type 2 diabetes. Diabetes Metab 2003; 29: 6S53-61.

26. Preiss D, Lloyd SM, Ford I, et al. Metformin for non-diabetic patients with coronary heart disease (the CAMERA study): a randomised controlled trial. Lancet Diabetes Endocrinol 2014; 2: 116-24.

27. Cariou B, Le Bras M, Langhi C, et al. Association between plasma PCSK9 and gamma-glutamyl transferase levels in diabetic patients. Atherosclerosis 2010; 211: 700-2.

28. Cui Q, Ju X, Yang T, et al. Serum PCSK9 is associated with multiple metabolic factors in a large Han Chinese population. Atherosclerosis 2010; 213: 632-6.

29. Yang SH, Li S, Zhang Y, et al. Positive correlation of plasma PCSK9 levels with HbA1c in patients with type 2 diabetes. Diabetes Metab Res Rev 2016; 32: 193-9.

30. Awan Z, Dubuc G, Faraj M, et al. The effect of insulin on circulating PCSK9 in postmenopausal obese women. Clin Biochem 2014; 47: 1033-9.

31. Brouwers MC, Troutt JS, van Greevenbroek MM, et al. Plasma proprotein convertase subtilisin kexin type 9 is not altered in subjects with impaired glucose metabolism and type 2 diabetes mellitus, but its relationship with non-HDL cholesterol and apolipoprotein B may be modified by type 2 diabetes mellitus: The CODAM study. Atherosclerosis 2011; 217: 263-7.

32. Costet P, Cariou B, Lambert G, et al. Hepatic PCSK9 expression is regulated by nutritional status via insulin and sterol regulatory element-binding protein $1 \mathrm{c}$. J Biol Chem 2006; 281: 6211-8.

33. Cariou B, Le Bras M, Langhi C, et al. Association between plasma PCSK9 and gammaglutamyltransferase levels in diabetic patients. Atherosclerosis 2010; 211: 700-2.

34. Arsenault BJ, Pelletier-Beaumont E, Alméras N, et al PCSK9 levels in abdominally obese men: association with cardiometabolic risk profile and effects of a oneyear lifestyle modification program. Atherosclerosis 2014; 236: 321-6.

35. Zang M, Zuccollo A, Hou X, et al. AMP-activated protein kinase is required for the lipid-lowering effect of metformin in insulin-resistant human HepG2 cells. J Biol Chem 2004; 279: 47898-905.

36. Hardie DG. Minireview: the AMP-activated protein kinase cascade: the key sensor of energy status. Endocrinology 2003; 144: 5179-83.

37. Yang SH, Xu RX, Cui CJ, et al. Liraglutide downregulates hepatic LDL receptor and PCSK9 expression in HepG2 cells and $\mathrm{db} / \mathrm{db}$ mice through a HNF-1a dependent mechanism. Cardiovasc Diabetol 2018; 17: 48.

38. Molusky MM, Hsieh J, Lee SX, et al. Metformin and AMP kinase activation increase expression of the sterol transporters ABCG5/8 (ATP-Binding Cassette Transporter G5/G8) with potential antiatherogenic consequences. Arterioscler Thromb Vasc Biol 2018; 38: 1493-503. 